\title{
DKA and new-onset type 1 diabetes in Brazilian children and adolescents during the COVID-19 pandemic
}

Thais Milioni Luciano'

http:///orcid.org/0000-0002-3826-4496

Mariana Peduti Halah'

http://orcid.org/0000-0002-9411-6105

Mariana Teresa Alves Sarti

http://orcid.org/0000-0002-1433-3995

Vitor Gonçalves Floriano²

http://orcid.org/00000-0002-8069-6348

Benedito Antônio Lopes da Fonseca²

http://orcid.org/0000-0003-3159-5687

Raphael Del Roio Liberatore Junior ${ }^{1}$

http://orcid.org/0000-0001-8893-2192

Sonir Rauber Antonini'

http:///orcid.org/0000-0003-4778-8803

\begin{abstract}
We assess the severity and frequency of diabetic ketoacidosis (DKA) in new-onset type 1 diabetes mellitus (T1D) patients and in patients with previous diagnosis ofT1D in a referral Brazilian university hospital in the first five months of the COVID-19 pandemic. We also compare the data with data from pre-pandemic periods. Forty-three new-onset T1D patients were diagnosed between April and August of the years 2017, 2018, 2019, and 2020. During the COVID-19 pandemic, the number of newonset T1D was over twice the number of new-onset T1D in the same period in the three previous years. All the 43 patients survived and are now on outpatient follow-up. We also compared the characteristics of the T1D patients hospitalized between April and August of the years 2017, 2018, and 2019 (32 hospitalizations) to the characteristics of the T1D patients hospitalized between April and August/2020 (35 hospitalizations; 1 patient was hospitalized twice in this period). Fourteen of the 34 patients admitted during the pandemic presented with COVID-19-related symptoms (any respiratory symptom, fever, nausea, vomiting, and diarrhea), but only one had positive SARS-CoV-2 RT-PCR test. Samples from 32 out of these 34 patients were assayed for SARS-CoV-2 antibodies, and four patients were positive for total antibodies ( $\lg M$ and $\lg G$ ). In agreement with recent reports from European countries, we observed increased frequency of DKA and severe DKA in new-onset and previously diagnosed T1D children and adolescents in a large referral public hospital in Brazil in the first five months of the COVID-19 pandemic. The reasons for this outcome might have been fear of SARSCoV-2 infection in emergency settings, the more limited availability of primary healthcare, and the lack of school personnel's attention toward children's general well-being.
\end{abstract}

Keywords

DKA; Type 1 diabetes; Sars-CoV-19; coronavirus pandemic
1 Divisão de Endocrinologia Pediátrica, Departamento de Pediatria, Faculdade de Medicina de Ribeirão Preto, Universidade de São Paulo, Ribeirão Preto, SP, Brasil 2 Divisão de Doenças Infecciosas, Departamento de Clínica Médica, Faculdade de Medicina de Ribeirão Preto, Universidade de São Paulo, Brasil
Correspondence to: Sonir Rauber Antonini Departamento de Pediatria Faculdade de Medicina de Ribeirão Preto, Universidade de São Paulo, Brasil

Av. Bandeirantes, 3900 14049-900 - Ribeirão Preto, SP, Brasil antonini@fmrp.usp.br

Received on Jan/26/2021 Accepted on Sept/22/2021

DOI: 10.20945/2359-3997000000433

\section{INTRODUCTION}

$\mathrm{T}$ The 2020 coronavirus pandemic is an unprecedented health emergency in modern times. While the demand for medical care by patients with acute respiratory syndrome caused by SARS-CoV-2 has increased in emergency settings, the search for medical care for other causes has declined steeply (1). Reports mainly from developed countries have speculated that the fear of COVID-19 contamination may have delayed the search for medical assistance, thereby aggravating the clinical conditions of patients with other diseases, such as type 1 diabetes mellitus (TID) (2). Herein we assess the severity and frequency of diabetic ketoacidosis (DKA) in new-onset type 1 diabetes mellitus (TID) patients and in patients with previous diagnosis of TID in a referral Brazilian university hospital in the first five months of the COVID-19 pandemic and compare these data with data from pre-pandemic periods. 


\section{MATERIALS AND METHODS}

This descriptive study collected data from the medical records of TID patients assisted at the Pediatric Endocrinology Service of the Ribeirao Preto Medical School University Hospital (University of São PauloBrazil). This hospital complex is part of the Brazilian publicly funded unified healthcare system known as SUS, and it is the tertiary referral center for a population of approximately 3.5 million in a regional geographical area in the state of Sao Paulo, Southeastern Brazil. In this region, around $70 \%$ of the population uses the public health system, whereas $30 \%$ is covered by private health insurance. This hospital is also the regional emergency and tertiary reference hospital for pediatric endocrinology.

Data from patients diagnosed with TID between April and August of the years 2017, 2019, and 2019 (pre-pandemic period) and between April and August/2020 (COVID-19 pandemic period) were obtained and analyzed. The number of new cases of TlD per 1,000 patients seen in the pediatric emergency department was calculated. The same months of 20172020 were analyzed to compare the incidence of TID in the same service in different years and to compare our data with the results of studies that used a similar period in other countries.

We also compared information from patients with previous diagnosis of TID that required hospitalization between October/2019 and February/2020 (prepandemic period) and between April and August/2020 (pandemic period). These months were was chosen because data were available from the institution.

The following data were investigated: number of new-onset TID, number of decompensated patients with previous diagnosis of TID, frequency of DKA in these patients, severity and time to recover from DKA, frequency of hypokalemia, age, sex, time elapsed since TID diagnosis, time elapsed since the last follow-up visit (for the patients with previously diagnosed TID), and weight $\mathrm{z}$-score. Comparisons were made between the pre-pandemic and pandemic periods. Symptomatic patients were submitted to RT-PCR for SARS-CoV-2, and the presence of SARS-CoV-2 antibodies was prospectively evaluated.

New-onset TID was defined by glycemia greater than or equal to $7 \mathrm{mmol} / \mathrm{L}(126 \mathrm{mg} / \mathrm{dL})$ in fasting plasma or greater than $11.1 \mathrm{mmol} / \mathrm{L}(200 \mathrm{mg} / \mathrm{dL})$ in a random measurement, associated with polyuria, polydipsia, polyphagia, and weight loss in previously healthy individuals aged less than 18 years, with or without DKA in the first presentation, and in need of using insulin to control hyperglycemia. Patients who were diagnosed before the age of 12 months, who had family history of diabetes before the age of 35 years over three generations, who had associated conditions suggesting the use of any medication, or who had a disease or syndrome associated with diabetes were not included. When clinical diagnosis was unclear, diabetes-associated autoantibodies (GAD and IA2) were collected. New-onset was considered when the diagnosis of TID, with or without DKA, was made between April and August of the years 2017, 2018, 2019, and 2020.

DKA was diagnosed in patient with glycemic equal or greater than $13.8 \mathrm{mmol} / \mathrm{L}(250 \mathrm{mg} / \mathrm{dL})$ and serum $\mathrm{pH}$ lower than 7.3 or serum bicarbonate lower than 15 $\mathrm{mmol} / \mathrm{L}$. Severe DKA was defined as serum $\mathrm{pH}$ lower than 7.1 or serum bicarbonate lower than $5 \mathrm{mEq} / \mathrm{L}$. Hypocalemia was defined by serum potassium levels lower than $3.5 \mathrm{mEq} / \mathrm{L}$. All the patients included in this analysis were first attended in the pediatric emergency department to confirm or to exclude DKA, and then they were transferred to the pediatric endocrinology ward.

The means, percentages, and variability were calculated, and the chi-squared test and Fisher's exact test were applied accordingly.

This study was registered in the Plataforma Brazil and the Ethics Committee of the Ribeirao Preto Medical School University Hospital approved this study under the CAAE protocol number 37130820.5.0000.5440.

\section{RESULTS}

Forty-three new-onset TID patients were diagnosed between April and August of the years 2017, 2018, 2019, and 2020 (Table 1). In the first five months of the COVID-19 pandemic, the number of new-onset TID was over twice the number of new-onset TID in the same period of the three previous years. All the 43 patients survived and are now on outpatient follow-up.

We also compared the characteristics of the TID patients hospitalized between April and August of the years 2017, 2018, and 2019 (pre-pandemic period, 32 hospitalizations) to the characteristics of the TID patients hospitalized between April and August/2020 (pandemic period, 35 hospitalizations; 1 patient was hospitalized twice in this period) (Table 2). 
Table 1. Characteristics of new-onset T1D patients from April to August of 2017, 2018, 2019, and 2020

\begin{tabular}{|c|c|c|c|c|}
\hline \multirow{2}{*}{ Clinical Features } & \multicolumn{4}{|c|}{ Year } \\
\hline & $2017(n=8)$ & $2018(n=9)$ & $2019(n=8)$ & $2020(n=18)$ \\
\hline New onset T1D cases/1,000 patients seen in pediatric emergency department & 5.2 & 5.4 & 4.1 & 9.9 \\
\hline $\begin{array}{l}\text { Age at diagnosis (years) } \\
\text { Median (range) }\end{array}$ & $\begin{array}{c}8.9 \\
(4.9-13)\end{array}$ & $\begin{array}{c}7.9 \\
(1.1-13.3)\end{array}$ & $\begin{array}{c}7.4 \\
(2.1-14.5)\end{array}$ & $\begin{array}{c}7.6 \\
(1.2-15.5)\end{array}$ \\
\hline \multicolumn{5}{|l|}{ Age (years) } \\
\hline$<6$ & 1 & 3 & 4 & 7 \\
\hline$\geq 6$ & 7 & 6 & 4 & 11 \\
\hline Female (n) & 4 & 5 & 2 & 9 \\
\hline $\begin{array}{l}\text { Weight Z-score } \\
\text { Median (range) }\end{array}$ & $\begin{array}{c}0.1 \\
(-2.3-2)\end{array}$ & $\begin{array}{c}-0.1 \\
(-2.4-1.6)\end{array}$ & $\begin{array}{c}0.6 \\
(-1.4-3.2)\end{array}$ & $\begin{array}{c}0.2 \\
(-4.6-2.9)\end{array}$ \\
\hline DKA at diagnosis (n) & 1 & 6 & 2 & 12 \\
\hline Severe DKA ${ }^{a}$ & 0 & 3 & 0 & 6 \\
\hline Frequency of hypokalemiab & 3 & 5 & 1 & 11 \\
\hline
\end{tabular}

DKA: diabetic ketoacidosis. ${ }^{a}$ Severe DKA: serum pH lower than 7.1 or serum bicarbonate lower than $5 \mathrm{mEq} / \mathrm{L} .{ }^{\mathrm{b}}$ Hypokalemia: plasma potassium levels lower than $3.5 \mathrm{mEg} / \mathrm{L}$.

Table 2. Characteristics of T1D inpatients in the pre-pandemic and pandemic periods

\begin{tabular}{|c|c|c|c|}
\hline \multirow{3}{*}{ Clinical Features } & \multicolumn{2}{|c|}{ Period } & \multirow{3}{*}{$\mathrm{p}$-value } \\
\hline & \multicolumn{2}{|c|}{$\mathbf{N}$} & \\
\hline & Pre-pandemic $(n=32)$ & Pandemic $(n=35)$ & \\
\hline Age at diagnosis (years) & 11.9 & 10.4 & * \\
\hline Median (range) & $(1.6-17)$ & $(1.25-18.6)$ & \\
\hline \multicolumn{4}{|l|}{ Age (years) } \\
\hline$<6$ & 2 & 7 & $p=0.09$ \\
\hline$\geq 6$ & 30 & 28 & \\
\hline Female (n) & 21 & 21 & $p=0.63$ \\
\hline Weight Z-score & -0.2 & -0.01 & * \\
\hline Median (range) & $(-5.1-2.2)$ & $(-4.6-2.9)$ & \\
\hline \multicolumn{4}{|l|}{ Hospitalization } \\
\hline New-onset T1D (n) & 6 & 18 & $\mathrm{p}=0.01$ \\
\hline Previous diagnoses & 26 & 17 & ** \\
\hline Time since T1D diagnosis (years). Median (range) & $5.7(2-13.7)$ & $3.1(2.7-16)$ & \\
\hline Time since last follow-up visit (months). Median (range) & $1.5(0.2-12)$ & $3(0.2-7.5)$ & \\
\hline \multicolumn{4}{|l|}{ DKA } \\
\hline New-onset T1D with DKA (n) & 5 & 12 & $\mathrm{p}=0.07$ \\
\hline New-onset T1D with severe DKA (n) & 2 & 9 & $p=0.03$ \\
\hline Total of DKA in hospitalizations (n) & 11 & 22 & $p=0.01$ \\
\hline Total severe DKA in hospitalizations (n) & 4 & 12 & $\mathrm{p}=0.03$ \\
\hline Hours for DKA resolution (hours) & 10.8 & 10.9 & * \\
\hline Median (range) & $(4-20)$ & $(2-34)$ & \\
\hline \multicolumn{4}{|l|}{ Electrolytic disturbance } \\
\hline Frequency of hypokalemiab & 9 & 16 & $p=0.46$ \\
\hline
\end{tabular}


Fourteen out of the 34 patients admitted during the pandemic presented with COVID-19-related symptoms (any respiratory symptom, fever, nausea, vomiting, and diarrhea), but only one had positive SARS-CoV-2 RTPCR test. Samples from 32 out of these 34 patients were assayed for SARS-CoV-2 antibodies, and four patients were positive for total antibodies ( $\operatorname{IgM}$ and $\mathrm{IgG})$. The patient with positive SARS-CoV-2 RT-PCR test was one of the four patients who tested positive for total antibodies but had no different hospitalization profile. Three of the latter four patients had previous diagnosis of TID; only one was diagnosed with newonset TID. The new-onset TID patient presented with DKA, but it was not severe. As for the three patients with previous diagnosis of TID, one had DKA, one had severe DKA, and one had decompensation without DKA, but their hospitalization profiles were not different compared to the other patients.

\section{DISCUSSION}

In the first five months of the COVID-19 pandemic, we observed higher frequency and severity of DKA in newonset TID children and adolescents, as well as a higher number of TID diagnosis in a large referral hospital in Southeastern Brazil. Similar findings were reported in Germany and London-UK $(3,4)$. A multicentric Italian report also noted increased severity and frequency of DKA, but the number of new-onset TID did not increase when compared to 2019 (5).

To date, there is no known relationship between the SARS-CoV-2 infection and the development of TID or DKA. However, it has been speculated that the number of new cases has increased during the pandemic. An association between SARS-CoV-2 and DKA, especially severe DKA, is not evident, either. Here, only one out of 14 patients submitted to RT-PCR tested positive for SARS-CoV-2. In addition, SARS-CoV-2 total antibodies were positive in this and three other patients (12\%). Thus, in most of our patients, DKA and TID cannot be attributed to COVID-19.

We believe that the increased frequency of DKA, especially severe DKA, during the pandemic period is related to delayed search for medical care due to fear of being contaminated with SARS-CoV-2 in emergency settings. The lower availability of primary healthcare might have contributed to this outcome. School closures may have been another reason for this finding given that teachers and school personnel were prevented from paying attention to students' well-being.

In agreement with recent reports from European countries, we also observed increased frequency of DKA and severe DKA in new-onset and previously diagnosed TID children and adolescents in a large referral public hospital in Brazil. Additionally, the number of T1D cases apparently increased, but this finding has yet to be confirmed by longer and more detailed observation.

Acknowledgement: we thank all the pediatric emergency and pediatric endocrinology teams, including the medical staff and residents who provided medical assistance for the patients included in this article.

Funding statement: this work was supported by the Medical Research Council FAPESP (2014/02989-6).

Disclosure: no potential conflicts of interest relevant to this article were reported.

\section{REFERENCES}

1. Scaramuzza A, Tagliaferri F, Bonetti L, Soliani M, Morotti F, Bellone $\mathrm{S}$, et al. Changing admission patterns in paediatric emergency departments during the COVID-19 pandemic. Arch Dis Child. 2020;105(7):704-6.

2. Baum A, Schwartz MD. Admissions to Veterans Affairs Hospitals for Emergency Conditions During the COVID-19 Pandemic. JAMA. 2020;324(1):96-9.

3. Kamrath C, Mönkemöller K, Biester T, Rohrer TR, Warncke K, Hammersen $\mathrm{J}$, et al. Ketoacidosis in Children and Adolescents With Newly Diagnosed Type 1 Diabetes During the COVID-19 Pandemic in Germany. JAMA. 2020;324(8):801-4.

4. Unsworth R, Wallace S, Oliver NS, Yeung S, Kshirsagar A, Naidu $\mathrm{H}$, et al. New-OnsetType 1 Diabetes in Children During COVID-19: Multicenter Regional Findings in the U.K. Diabetes Care. 2020;43(11):e170-1.

5. Rabbone I, Schiaffini R, Cherubini V, Maffeis C, Scaramuzza A; Diabetes Study Group of the Italian Society for Pediatric Endocrinology and Diabetes. Has COVID-19 Delayed the Diagnosis and Worsened the Presentation of Type 1 Diabetes in Children? Diabetes Care. 2020 Nov;43(11):2870-2. 